\title{
Use of the Google Drive Platform By University Students
}

\section{Utilización de la Plataforma Google Drive en Estudiantes Universitarios}

\author{
Edgar Rolando Morales Caluñaa ${ }^{1}$ and Fernando Xavier Altamirano Capelo \\ ${ }^{1}$ Facultad de Contabilidad y Auditoria, Universidad Técnica de Ambato, Ambato, Ecuador \\ ${ }^{2}$ Facultad de Salud Pública, Escuela Superior Politécnica de Chimborazo, Riobamba, Ecuador
}

XVIII International Seminar on Health, Food and Human Nutrition

Corresponding Author:

Edgar Rolando Morales

Caluñaa

er.morales@uta.edu.ec

Published: 9 September 2021

Production and Hosting by

Knowledge $\mathrm{E}$

(c) Edgar Rolando Morales Caluñaa and Fernando Xavier Altamirano Capelo. This article is distributed under the terms of the Creative Commons Attribution License, which permits unrestricted use and redistribution provided that the original author and source are credited.

\section{Abstract}

This paper presents the level of post-use of the Google Drive platform in academic and personal activities in university students. A descriptive research was carried out on students who attended the TICs between the semesters March-August 2013 and April-August 2016 at the School of Public Health of the Politecnica Superior School of Chimborazo, through the application of an online questionnaire. With 87 valid responses, $62.1 \%$ use the platform in personal activities, $88.5 \%$ use files and folders, the most used tool is $62.1 \%$, while $65.5 \%$ Synchronized your smart phone with Google Drive. Students who participated in the study continue to use Google Drive in their academic and personal activities.

Keywords: collaborative work, cloud computing, mobile devices, Google Drive.

\section{Resumen}

Este trabajo presenta el nivel de pos-utilización de la plataforma Google Drive en actividades académicas y personales en estudiantes universitarios. Se desarrolló una investigación de tipo descriptivo en estudiantes que cursaron las cátedras TICs entre los semestres Marzo-Agosto 2013 y Abril-Agosto 2016 en la Facultad de Salud Pública de la Escuela Superior Politécnica de Chimborazo, mediante la aplicación de un cuestionario en linea. Con 87 respuestas válidas, el $62,1 \%$ utiliza la plataforma en actividades personales, el 88,5\% utiliza para compartir archivos y carpetas, la herramienta más utilizada es el formulario con el 62,1\%, mientras que el 65,5\% tiene sincronizado su celular inteligente con Google Drive. Los estudiantes que participaron en el estudio continuan utilizando Google Drive en sus actividades académicas y personales.

Palabras Clave: trabajo colaborativo, computación en la nube, dispositivos móviles, Google Drive.

\section{Introducción}

Las cátedras del área básica, relacionadas a las Tecnologías de la Información y Comunicación (TICs) de la Facultad de Salud Pública contribuye al desarrollo de capacidades y destrezas para una correcta utilización de herramientas informáticas, tomando en S OPEN ACCESS y profesional de las personas en todas las áreas y profesiones [1]. 
Los programas analíticos de las cátedras TICs, están basados en contenidos relacionados al procesamiento de la información bajo el paradigma de la Computación en la Nube [2] y en la utilización de software específico para cada carrera.

Uno de los tópicos que se estudian, está relacionado a la gestión de la información en línea como almacenamiento, trabajo colaborativo, ofimática en línea entre otros, con la finalidad que el estudiante disponga de los conocimientos y habilidades necesarias para gestionar la información generada en otras cátedras y de esta manera mejorar el proceso de aprendizaje [3].

Con el advenimiento del internet, las fuentes tradicionales de información como libros, revistas, entre otros, en formato físico han sido desplazados por los repositorios digitales [4], motores de búsqueda [5], también debe ser considerado como una justificación relevante para que la formación académica de los estudiantes se base en una sólida formación en el manejo de herramientas informáticas.

El objetivo del presente artículo es conocer el nivel de pos-utilización de la plataforma Google Drive por los estudiantes de la Facultad de Salud Pública de la Escuela Superior Politécnica de Chimborazo, entre los periodos Marzo - Julio 2013 y Abril-Agosto 2016.

\subsection{Pensum de estudios}

Se entenderá por pensum de estudios todas aquellas materias que conforman los cursos dentro de una organización, el pensum de estudios podrá incrementarse dependiendo de las necesidades de la entidad [6]

Los pensum de estudios [7] están diseñados en forma matricial, donde las columnas contienen los semestres o niveles de la carrera, mientras en las filas se presentan los diferentes campos de formación del futuro profesional, entre los cuales están: Fundamento Teórico, Praxis Profesional [8], Epistemología y Metodología de Investigación, Comunicación y Lenguaje, Integración de Saberes, Contextos y Cultura. Generalmente en carreras relacionadas a la salud, las cátedras TICs se ubicaban dentro del Área Básica [9] y actualmente en el campo Comunicación y Lenguaje.

Los principales temas que contienen los Programas de Estudios de Asignatura (PEA) [10], constan manejos de procesadores de textos, gestores bibliográficos, hojas de cálculo, manejos de formularios para la generación de encuestas, trabajo colaborativo, sincronización de la información, mediante la utilización de la plataforma Google Drive [11].

\subsection{Computación en la nube}

La computación en la nube [12] o 'Cloud Computing' es un nuevo mecanismo de procesamiento de la información en el Internet, esta revolucionaria forma de gestión de la información ofrece a los usuarios servicios y ventajas como almacenamiento en línea, ofimática en línea, transparencia en la utilización de equipos hardware, integración con servicios de terceros entre otras [13]. 
Las plataformas 'Cloud Computing', comercialmente se desarrollan en dos frentes, la primera alternativa bajo una política de libre utilización, limitada especialmente por la capacidad de almacenamiento, como Google Drive y OneDrive [14], mientras que la segunda alternativa está orientada a la venta de servicios bajo demanda, como Windows Azurre, Google App Engine entre otros [15].

Las plataformas de libre acceso disponen de servicios que pueden adaptar a los procesos educativos para mejorar la educación en todos los niveles. La literatura existente relacionada a la utilización de estas plataformas en el proceso de enseñanza - Aprendizaje destaca estudios como: Trabajo en equipo con Google Drive en la universidad online [16], donde destaca la facilidad de manejo, la capacidad de trabajo en tiempo real a distancia, así como también la utilidad pedagógica en la educación, mientras que en el estudio Una experiencia personal: Google Drive para el portafolio virtual docente-estudiante en la docencia universitaria [17], se concluye que Google Drive es una buena alternativa para la gestión del portafolio virtual para estudiantes y docentes [18].

También refleja la utilización de este tipo de plataformas para el diseño y dictado de cátedras, en el estudio Enseñanza de Photoshop mediante Youtube y Google Drive [19], se concluye que la utilización de este tipo de plataformas genera un mejor acceso a la información, participación del alumnado y seguimiento de actividades.

\subsection{Características de la computación en la nube}

Entre las principales características que brinda la computación en la nube a los usuarios destacan:

- Flexibilidad para compartir información.

- Interfaces amigables.

- Capacidad para generar espacios de trabajo colaborativo.

- Independencia del sistema operativo de computador.

- Requerimientos mínimos en hardware y software del computador.

- Capacidad de acceso a la información desde distintos puntos y medios.

- Integración con servicios de terceros, y otras.

Generalmente estas plataformas disponen de varios servicios que los usuarios pueden utilizar y configurar de acuerdo con las necesidades.

\subsection{Google Drive}

La utilización de la plataforma Google Drive, en el ejercicio de la docencia universitaria ha permitido generar diferentes artículos científicos [17, 20], relacionados a incorporación de esta plataforma como herramienta complementaria, además de la producción del libro titulado 'Computación en la Nube con Google Drive' [21], donde se menciona las principales funcionalidades de esta plataforma. 
La capacidad de gestionar la información generada por los estudiantes directamente en internet, simulando un disco duro virtual, donde los estudiantes pueden almacenar la información a base de directorios, la posibilidad de compartir con otros usuarios espacios virtuales para la generación de trabajo colaborativo [22] hacen de Google Drive una alternativa válida para la utilización en los distintos procesos de aprendizaje.

En la actualidad el término 'Trabajo Colaborativo' [23], ha tomado fuerza dentro de los procesos de aprendizaje en la educación universitaria, con la finalidad de generar nuevas alternativas para que los estudiantes realicen las actividades asignadas por los docentes de una forma no presencial, los servicios de Google Drive permiten implementar espacios de trabajo colaborativo mediante la utilización de directorios y agregando a los usuarios mediante cuentas de correo electrónico de Gmail. La herramienta permite generar espacios de 'chat', notas por cada uno de los elementos generados en el espacio de trabajo colaborativo [24].

Otra de las características de Google Drive, es la facilidad de uso [16], Google Drive, está diseñado a base de interfaces amigables e intuitivas que no representan un problema al momento de utilizar la herramienta, al contrario estudios afirman que existe un desconocimiento de la existencia de esta plataforma [25], partiendo de esta evidencia, es necesario generar políticas que permitan socializar a las comunidades universitarias los beneficios de la utilización de Google Drive en el proceso educativo y en las actividades profesionales de los estudiantes.

\subsection{Materiales y Métodos}

Se desarrolló una investigación de tipo descriptivo [26] a partir de la aplicación de una encuesta a los estudiantes de las carreras de Medicina y Nutrición y Dietética en los semestres comprendidos entre Marzo-Julio 2013 y Abril-Agosto 2016, en la Facultad de Salud Pública de la Escuela Superior Politécnica de Chimborazo. La encuesta se difundió mediante correos electrónicos y redes sociales, se obtuvieron un total de 87 respuestas válidas, no se aplicó ninguna técnica de muestreo.

El instrumento de la toma de datos se desarrolló expresamente para esta investigación, datos sociodemográficos [27] ocho ítems, mientras que los ítems relacionados al uso de la plataforma Google Drive ocho ítems, formado por preguntas [28] abiertas, cerradas de selección múltiple, opción múltiple y escala Likert. El cuestionario se construyó en la Herramienta Formularios de Google Drive [29].

Los datos se procesaron utilizando las Hojas de Cálculos de Google Drive y el reporte de datos estadísticos generados por la herramienta donde se construyó el instrumento, las preguntas abiertas se procesaron mediante el análisis estadístico de datos textuales AEDT [30], en la página Web 2.0 Textalyser, disponible en: http://textalyser.net/, dos profesionales del área validaron el cuestionario antes de su aplicación. 


\section{Resultados}

La ofimática en línea es uno de los principales servicios que ofrece la plataforma Google Drive, en el presente estudio, el nivel de utilización mediante la alternativa 'Mucho', la herramienta formularios con un 62,1\% lidera el nivel de utilización de los encuestados, seguido de la herramienta procesador de textos con un $56,3 \%$, presentaciones interactivas $54,0 \%$, hojas de cálculo $51,7 \%$ y complementos el $47,1 \%$. La herramienta formularios permite generar cuestionarios de forma fácil y eficiente por tal motivo los estudiantes utilizan en el desarrollo de las actividades académicas.

OFIMÁTICA EN LINEA

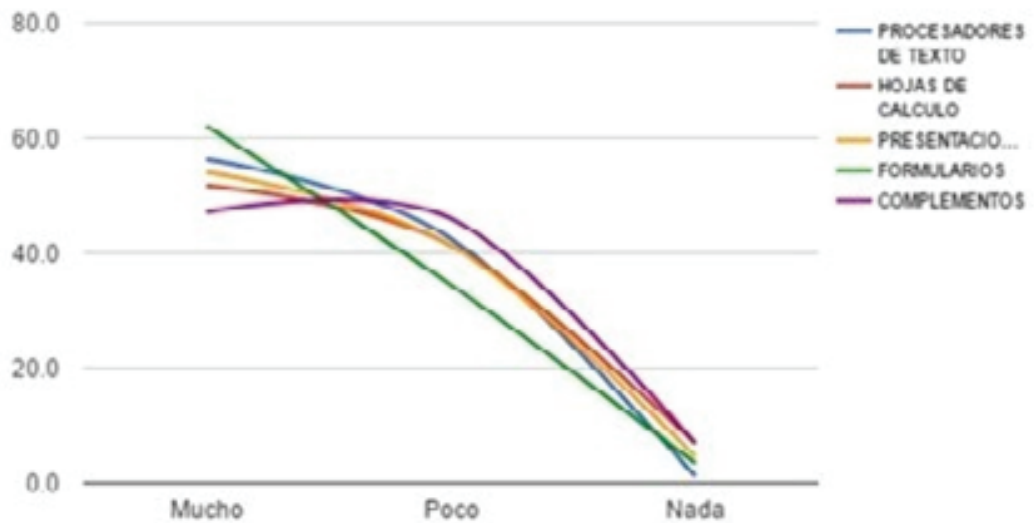

Figure 1

Nivel de utilización de ofimática en línea en la plataforma Google Drive.

El $88,5 \%$ de los encuestados, utiliza la alternativa de trabajo colaborativo, para compartir archivos y carpetas en las actividades académicas, gestionar la información bajo este paradigma, permite a los estudiantes organizar de una manera adecuada sus recursos como tiempo y dinero, el 70,1\% utiliza para la creación de espacios de trabajo.

El 63,2\% utiliza la alternativa para el seguimiento de las tareas asignadas dentro de los grupos de trabajo, el 51,7\% utiliza 'chat' en la realización de las actividades relacionadas al trabajo colaborativo. La media de la opción 'Mucho' en este apartado es de $68,4 \%$.

El principal objetivo del presente estudio es conocer la pos-utilización que hacen los estudiantes a la plataforma en otras cátedras, en actividades personales, laborales y el interés por profundizar los conocimientos acerca de la herramienta. En la Tabla 1 se detallan los valores de pos-utilización de la herramienta.

Otra de las potencialidades de Google Drive es la sincronización de la información en otros dispositivos como computador de escritorio, portátiles, celulares inteligentes, en la presente encuesta el $65,5 \%$ ha sincronizado Google Drive con el celular, equipos portátiles el $54 \%$ y únicamente el 4,6 de los encuestados no a sincronizado con algún dispositivo móvil.

Finalmente, en la pregunta acerca de las debilidades y fortalezas de Google Drive, con un corpus creado con una base de 5 caracteres, se obtuvo un total de 543 palabras, 
TRABAJO COLABORATIVO

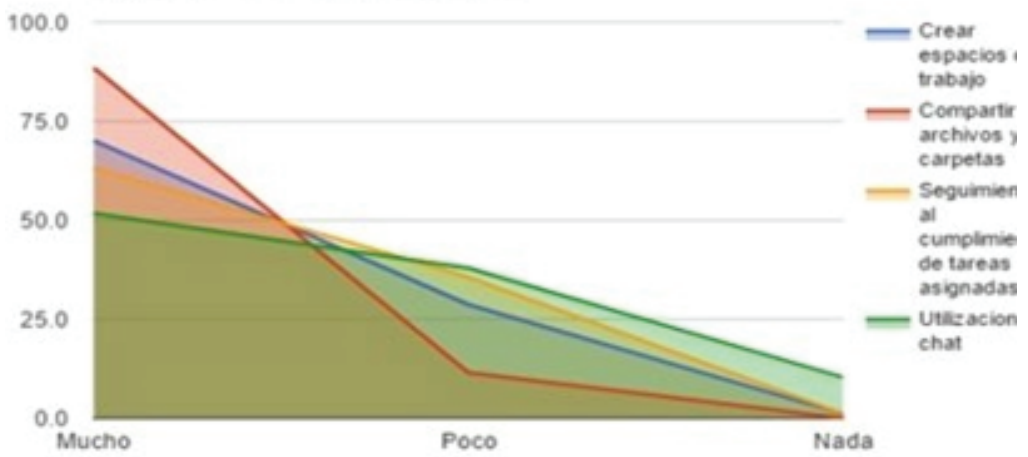

Figure 2

Nivel de utilización de las opciones de trabajo colaborativo en Google Drive.

Table 1

Pos-utilización de la plataforma Google Drive.

\begin{tabular}{lr}
\hline Actividades & Utilización (Mucho) \\
Actividades laborales & $52,90 \%$ \\
\hline Actividades personales & $62,10 \%$ \\
\hline Interés por profundizar en el tema & $47,10 \%$ \\
Utilización en otras cátedras & $59,80 \%$ \\
\hline
\end{tabular}

Fuente: Encuesta aplicada.

270 palabras distintas que corresponden al 49,7\% de densidad léxica. Se obtuvieron las 10 primeras palabras, la Tabla 2 detalla el orden de las palabras.

Las palabras están relacionadas a las actividades que los usuarios realizan en la plataforma Google Drive, esto permite afirmar que los encuestados están involucrados con la utilización de la plataforma. En base las palabras con mayor frecuencia de repetición, la Tabla 3 contiene las frases relevantes acerca de las fortalezas y debilidades de Google Drive.

\section{Conclusiones}

Los estudiantes demuestran la pos-utilización de Google Drive en sus actividades académicas y personales, en general las opiniones son aceptables en los diferentes aspectos consultados, estos resultados demuestran la importancia de la inclusión de cátedras relacionadas a las TICs en la malla curricular de las diferentes carreras para la formación profesional de los estudiantes. 
Table 2

Diez palabras del análisis estadístico de datos textuales.

\begin{tabular}{rlrrr}
\hline Orden & Palabra & Ocurrencias & Frecuencia & Rango \\
\hline $\mathbf{1}$ & documentos & 14 & $3.20 \%$ & 1 \\
$\mathbf{2}$ & permite & 13 & $3 \%$ & 2 \\
\hline $\mathbf{3}$ & información & 11 & $2.50 \%$ & 3 \\
\hline $\mathbf{4}$ & archivos & 11 & $2.50 \%$ & 3 \\
\hline $\mathbf{5}$ & almacenamiento & & $2.30 \%$ & 4 \\
\hline $\mathbf{6}$ & debilidad & 9 & $2.10 \%$ & 5 \\
\hline $\mathbf{7}$ & almacenar & 9 & $2.10 \%$ & 5 \\
\hline $\mathbf{8}$ & internet & 8 & $1.80 \%$ & 6 \\
\hline $\mathbf{9}$ & cualquier & 8 & $1.80 \%$ & 6 \\
\hline $\mathbf{1 0}$ & compartir & 8 & $1.80 \%$ & 6
\end{tabular}

Fuente: Textalyser.

Table 3

Fortalezas y debilidades de Google Drive indicadas por los encuestados.

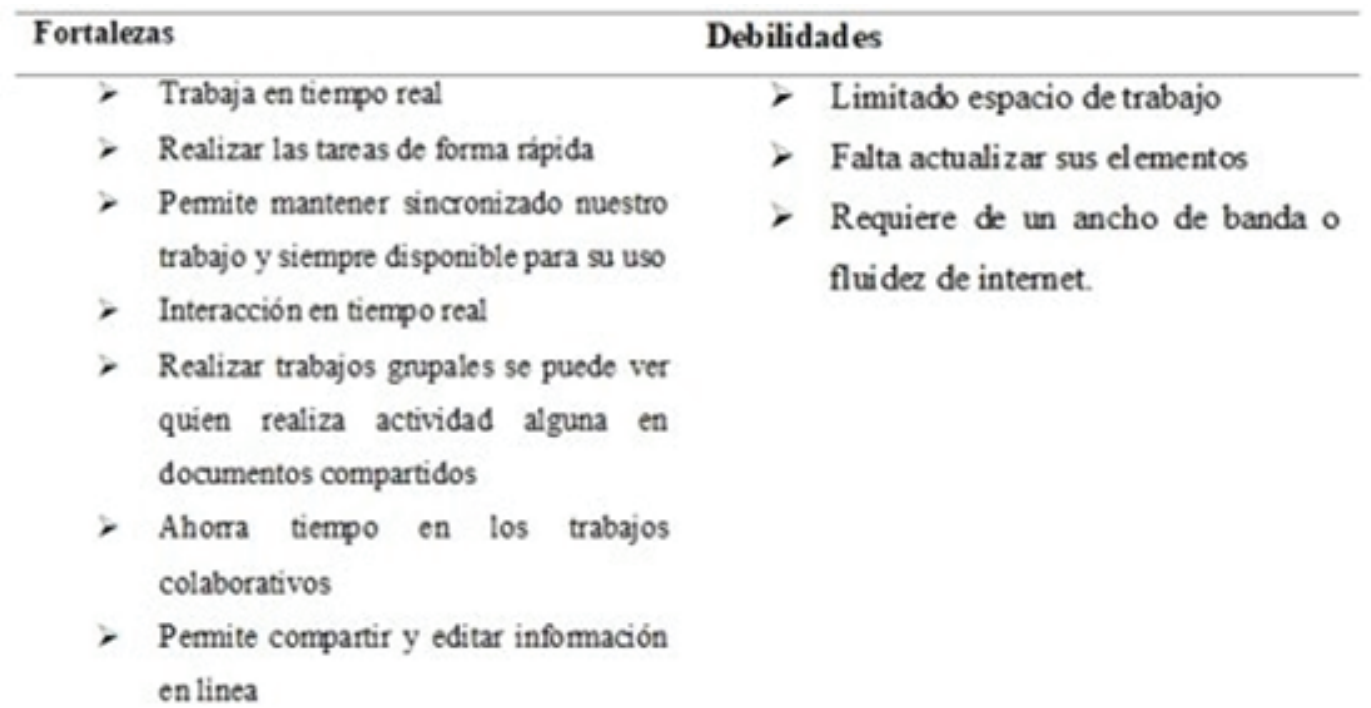

Fuente: Base de datos encuestas aplicadas.

Fomentar la utilización de Google Drive en el proceso educativo, permite a los estudiantes contar con nuevas alternativas para realizar sus trabajos académicos y gestionar adecuadamente la información.

Los servicios de ofimática en línea y trabajo colaborativo son alterativas innovadoras de utilización en docentes y estudiantes con el fin de ahorrar recursos como económicos y materiales en el desarrollo de las actividades académicas. 
La sincronización de la información en diferentes dispositivos permite a los usuarios interactuar desde cualquier momento y lugar con la información almacenada en Google Drive.

Es necesario desarrollar políticas de seguimiento para conocer el nivel de posutilización de los conocimientos impartidos en las diferentes cátedras.

\section{References}

[1] Huatuco RM, Velásquez WL. El uso de las TIC en la enseñanza profesional. Ind Data. 2009;12(2):61-67.

[2] Martín RL. Educación y entorno territorial de la Universitat de València: Conferencias impartidas en el Programa Universitat i Territori. Universitat de València; 2014. 280 p.

[3] Díaz JT, Gómez JIA. Desarrollo profesional docente ante los nuevos retos de las tecnologías de la información y la comunicación en los centros educativos. Píxel-Bit Rev Medios Educ. 2009;(34):31-47.

[4] Martínez AC, Conde ER. Participación y visibilidad web de los repositorios digitales universitarios en el contexto europeo. Comunicar. 2013;20(40):193-201.

[5] Saura JR, Palos-Sánchez P, Navalpotro FD. El problema de la Reputación Online y Motores de Búsqueda: Derecho al Olvido. Cad Dereito Actual. 2018;(8):221-229.

[6] Principio de Administración. ITM; 2008. 143 p.

[7] Antoniene JIG, Durán GIN, Pinto MA. La educación universitaria: Una mirada desde la complejidad. Secr Educ JALISCO. 2017;46.

[8] Sandoval LEP, Prado ALR. Relación entre teoría y praxis en la formación de profesionales de enfermería: Revisión integradora de literatura. Enferm Glob. 2017;16(47):679-706.

[9] Escuela Superior Politécnica De Chimborazo, Facultad De Salud Publica Nutricion Y Dietetica. Mapa curricular escuela de nutrición y ditética. Disponible en: https://www.espoch.edu.ec/images/facultades/ salud_publica/archivos/PENSUM_END.pdf

[10] Rodríguez RG, García JC. Material didáctico para la asignatura Medicina General Integral. Rev Cuba Med Gen Integral. 2017;33(2):209-217.

[11] Ramos J. Productividad en la nube. XinXii; 2017. 40 p.

[12] Arias Á. Computación en la nube: 2a Edición. IT Campus Academy; 2015. 86 p.

[13] Silvia P. Para saber más. Colombia: Penguin Random House Grupo Editorial Colombia; 2011. 361 p.

[14] Smolinski G. Cloud watching: Un repaso de los mejores programas de almacenamiento en la nube. Babelcube Inc.; 2016. 23 p.

[15] Martín A, Chávez SB, Rodríguez NR, Valenzuela A, Murazzo MA. Bases de datos NoSQL en cloud computing. Paper presented at: XV Workshop de Investigadores en Ciencias de la Computación. 2013.

[16] Sánchez AC, De la Muela A. Trabajo en equipo con Google Drive en la universidad online. Innov Educ México DF. 2013;13(63):75-94.

[17] Caluña ERM. Una experiencia personal: Google Drive para el portafolio virtual docente-estudiante en la docencia universitaria. Rev Tecnológica - ESPOL. 2015;28(2).

[18] Perea LEV. Desarrollo de Habilidades en el uso de las Tecnologías de la Información y la Comunicacion: DHTIC. Luis Enrique Velazquez Perea; 2016. 103 p.

[19] Zamora JO. Enseñanza de Photoshop mediante Youtube y Google Drive. Available from: https://web. ua.es/es/ice/jornadas-redes-2014/documentos/comunicaciones-posters/tema-1/393251.pdf

[20] Caluña ERM. Portafolio docente con google drive. Rev Telem Tica. 2016;15(2):91-96.

[21] Morales E, Altamirano X. Computación en la Nube con Google Drive. Riobamba: La Caracola Editores; 2016. $124 \mathrm{p}$.

[22] Unid ED, Telmex A. Antología de competencias digitales. Editorial Digital UNID; 2015. 610 p.

[23] Frutos AE. Interculturalidad, mediación y trabajo colaborativo. Narcea Ediciones; 2016. 195 p.

[24] Benito VD, Villaverde VA, Franco MR, Muñoz RC. Google Docs: una experiencia de trabajo colaborativo desde las aulas universitarias: EN Metodologías de aprendizaje colaborativo a través de las tecnologías. Ediciones Universidad de Salamanca; 2014. 25 p.

[25] Baiges EB, Surroca NV. Valoración del uso de las herramientas colaborativas wikispaces y google drive, en la educación superior. Edutec Rev Electrónica Tecnol Educ. 2015;(49).

[26] Tamayo MT. El proceso de la investigación científica. Editorial Limusa; 2004. 440 p.

[27] Moreira MA, Salvat BG, García-Quismondo MÁM. Alfabetizaciones y tecnologías de la información y la comunicación. Síntesis; 2008. 
[28] Abascal E, Esteban IG. Análisis de encuestas. Esic Editorial; 2005. Disponible en: https://books.google. com.ec/ 